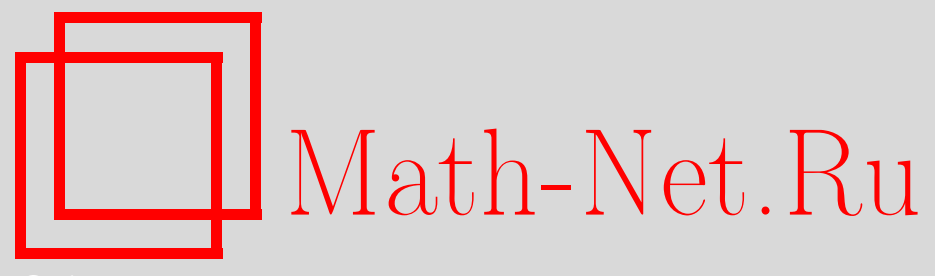

О. А. Репин, А. В. Тарасенко, Об одной задаче для уравнения смешанного типа с частной дробной производной Римана-Лиувилля, Вестн. Сам. гос. техн. ун-та. Сер. Физ.-мат. науки, 2016, номер 4, 636-643

DOI: https://doi.org/10.14498/vsgtu1516

Использование Общероссийского математического портала MathNet.Ru подразумевает, что вы прочитали и согласны с пользовательским соглашением

http://www.mathnet.ru/rus/agreement

Параметры загрузки:

IP : 54.81 .137 .203

26 апреля 2023 г., 09:01:42

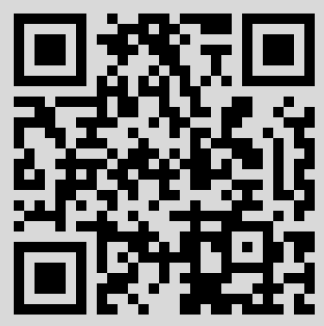


УДК 517.956.6

\title{
ОБ ОДНОЙ ЗАДАЧЕ ДЛЯ УРАВНЕНИЯ СМЕШАННОГО ТИПА С ЧАСТНОЙ ДРОБНОЙ ПРОИЗВОДНОЙ РИМАНА-ЛИУВИЛЛЯ
}

\author{
O. А. Репин ${ }^{1,2}$, А. В. Тарасенко ${ }^{2}$ \\ 1 Самарский государственный экономический университет, \\ Россия, 443090, Самара, ул. Советской Армии, 141. \\ 2 Самарский государственный технический университет, \\ Россия, 443100, Самара, ул. Молодогвардейская, 244.
}

\section{Аннотация}

Для уравнения смешанного типа, содержащего уравнение диффузии дробного порядка, доказаны единственность и существование решения нелокальной задачи, краевое условие которой представляет собой линейную комбинацию обобщенных операторов дробного порядка с гипергеометрической функцией Гаусса. Приведено явное решение исследуемой задачи.

Ключевые слова: краевая задача, обобщенный оператор дробного интегро-дифференцирования, дифференциальное уравнение дробного порядка, гипергеометрическая функция Гаусса.

\section{1. Введение. Рассмотрим уравнение}

$$
\begin{cases}u_{x x}-D_{0+, y}^{\alpha_{0}} u=0, & y>0,0<\alpha_{0}<1, \\ (-y)^{m} u_{x x}-u_{y y}+a_{0}(-y)^{\frac{m}{2}-1} u_{x}=0, & y<0,\end{cases}
$$

где $a_{0}$ - вещественная постоянная, $m>2$ в конечной области $\Omega$, ограниченной отрезками $A A_{0}, B B_{0}, A_{0} B_{0}$ прямых $x=0, x=1, y=1$ соответственно, лежащих в полуплоскости $y>0$, и его характеристиками

$$
A C: x-\frac{2}{m+2}(-y)^{\frac{m+2}{2}}=0, \quad B C: x+\frac{2}{m+2}(-y)^{\frac{m+2}{2}}=1
$$

в полуплоскости $y<0$. Здесь $D_{0+, y}^{\alpha_{0}}-$ частная дробная производная РиманаЛиувилля [1, с. 33].

Пусть $\Omega_{1}=\Omega \cap(y>0), \Omega_{2}=\Omega \cap(y<0), I \equiv A B-$ единичный интервал $0<x<1$ прямой $y=0$.

(C) 2016 Самарский государственный технический университет.

\section{Образец для цитирования}

Р еп и н О. А., Т а р а с ен к о А. В. Об одной задаче для уравнения смешанного типа с частной дробной производной Римана-Лиувилля // Вестн. Сам. гос. техн. ун-та. Сер. Физ.мат. науки, 2016. Т. 20, № 4. C. 636-643. doi: 10.14498/vsgtu1516.

\section{Сведения об авторах}

Олег Александрович Репин (д.ф.-м.н., проф.; matstat@mail.ru), заведующий кафедрой, каф. математической статистики и эконометрики ${ }^{1}$; профессор, каф. прикладной математики и информатики ${ }^{2}$.

Анна Валеръевна Тарасенко (к.ф.-м.н., доц.; tarasenko.a.v@mail.ru; автор, ведущий переписку), доцент, каф. высшей математики АСИ СамГТУ. 
ЗАДАчА. Найти решение $u(x, y)$ уравнения $(1)$ в области $\Omega$, удовлетворяющее краевым условиям

$$
u(0, y)=\varphi_{1}(y), \quad u(1, y)=\varphi_{2}(y), \quad 0 \leqslant y \leqslant 1
$$

$$
\begin{aligned}
A\left(I_{0+}^{a, b, \beta-1-a} u\left[\Theta_{0}(t)\right]\right) & (x)+B\left(I_{0+}^{a_{1}, a+b+\alpha-a_{1}, \beta-a-1} u_{y}(t, 0)\right)(x)+ \\
& +C\left(I_{0+}^{a_{2}, a+b+\alpha-a_{2}, \beta-a-1} u_{y}(t, 0)\right)(x)=g(x), \quad x \in I,
\end{aligned}
$$

а также условиям сопряюения

$$
\begin{gathered}
\lim _{y \rightarrow+0} y^{1-\alpha_{0}} u(x, y)=\lim _{y \rightarrow-0} u(x, y), \quad x \in \bar{I}, \\
\lim _{y \rightarrow+0} y^{1-\alpha_{0}}\left(y^{1-\alpha_{0}} u(x, y)\right)_{y}=\lim _{y \rightarrow-0} u_{y}(x, y), \quad x \in I .
\end{gathered}
$$

$3 \partial e c b$

$\alpha=\frac{m-2 a_{0}}{2(m+2)}, \beta=\frac{m+2 a_{0}}{2(m+2)}, \alpha+\beta=\frac{m}{m+2} ; \quad \Theta_{0}(x)=\frac{x}{2}-i\left[\frac{(m+2) x}{4}\right]^{\frac{2}{m+2}}$

- точка пересечения характеристик уравнения (1), выходящих из точек $(x, 0)(x \in I)$ с характеристикой $A C ;\left(I_{0+}^{\alpha, \beta, \eta} f\right)(x)$-оператор обобщенного дробного интегро-дифференцирования, введенный японским математиком M. Сайго [2] (см. такэе [1, с. 326-327], [3, с. 14], [4, с. 12]); A, B, C-вещественные константы, такие, что

$$
A<0, \quad B \geqslant 0, \quad C \geqslant 0 \quad \text { uли } \quad A>0, \quad B \leqslant 0, \quad C \geqslant 0
$$

$a, b, a_{1}, a_{2}$ - действительные числа, причем

$$
a>\max \{-\alpha, \beta-1\}, \quad 1+a+\alpha-2 \beta<a_{1}<a_{2}<2+a+\alpha
$$

$y^{1-\alpha_{0}} \varphi_{1}(y), y^{1-\alpha_{0}} \varphi_{2}(y) \in C([0,1]) ; \varphi_{1}(0)=\varphi_{2}(0)=0 ; g(x) \in C^{2}([0,1])$.

Решение поставленной задачи будем искать в классе дважды дифференцируемых в области $\Omega$ функций $u(x, y)$ таких, что

$$
\begin{gathered}
y^{1-\alpha_{0}} u(x, y) \in C\left(\bar{\Omega}_{1}\right), \quad u(x, y) \in C\left(\bar{\Omega}_{2}\right), \\
y^{1-\alpha_{0}}\left(y^{1-\alpha_{0}} u_{y}\right)_{y} \in C\left(\Omega_{1} \cup\{(x, y): 0<x<1, y=0\}\right), \\
u_{x x} \in C\left(\Omega_{1} \cup \Omega_{2}\right), \quad u_{y y} \in C\left(\bar{\Omega}_{2}\right) .
\end{gathered}
$$

Отметим, что исследуемая задача является продолжением работ $[5,6]$.

2. Единственность решения задачи. Пусть существует решение исследуемой задачи. Введем обозначения

$$
\begin{gathered}
\lim _{y \rightarrow 0+} y^{1-\alpha_{0}} u(x, y)=\tau_{1}(x), \quad \lim _{y \rightarrow 0-} u(x, y)=\tau_{2}(x) \\
\lim _{y \rightarrow 0+} y^{1-\alpha_{0}}\left(y^{1-\alpha_{0}} u(x, y)\right)_{y}=\nu_{1}(x), \quad \lim _{y \rightarrow 0-} u_{y}(x, y)=\nu_{2}(x) .
\end{gathered}
$$


Известно [7,8], что решение уравнения (1) в области $\Omega_{1}$, удовлетворяющее условию (2) и условию

$$
\lim _{y \rightarrow 0+} y^{1-\alpha_{0}} u(x, y)=\tau_{1}(x) \quad(0 \leqslant x \leqslant 1)
$$

дается формулой

$$
u(x, y)=\left.\int_{0}^{y} \frac{\partial G}{\partial \xi}\right|_{\xi=0} \varphi_{1}(\eta) d \eta-\left.\int_{0}^{y} \frac{\partial G}{\partial \xi}\right|_{\xi=1} \varphi_{2}(\eta) d \eta-\int_{0}^{1} G(x, y ; \xi, 0) \tau_{1}(\xi) d \xi
$$

где

$$
\begin{gathered}
G(x, y ; \xi, \eta)=\frac{\Gamma\left(\alpha_{0}\right)}{2}(y-\eta)^{\delta-1} \sum_{n=-\infty}^{\infty}\left[e_{1, \delta}^{1, \delta}\left(-\frac{|x-\xi+2 n|}{(y-\eta)^{\delta}}\right)-\right. \\
\left.-e_{1, \delta}^{1, \delta}\left(-\frac{|x+\xi+2 n|}{(y-\eta)^{\delta}}\right)\right], \\
e_{\alpha_{0}, \beta_{0}}^{\mu, \delta}(z)=\sum_{k=0}^{\infty} \frac{z^{k}}{\Gamma\left(\mu+k \alpha_{0}\right) \Gamma\left(\delta-k \beta_{0}\right)}, \quad \alpha_{0}>\beta_{0}, \quad \delta=\frac{\alpha_{0}}{2}, \quad \alpha_{0}>0, \\
e_{1, \delta}^{1, \delta}(z)=\sum_{k=0}^{\infty} \frac{z^{k}}{k ! \Gamma(\delta-\delta k)}, \quad \delta<1 .
\end{gathered}
$$

ЗАмечание. Решение $u(x, y)$ может быть выражено в терминах специальной функции Райта [7]

$$
\varphi(\gamma, \delta ; z)=\sum_{k=0}^{\infty} \frac{z^{k}}{k ! \Gamma(\delta+\gamma k)}
$$

поскольку

$$
e_{1, \frac{\alpha_{0}}{2}}^{1, \frac{\alpha_{0}}{2}}(z)=\varphi\left(-\frac{\alpha_{0}}{2}, \frac{\alpha_{0}}{2} ; z\right)
$$

Функциональное соотношение между $\tau_{1}(x)$ и $\nu_{1}(x)$, принесенное из параболической части $\Omega_{1}$ на линию $y=0$, имеет вид (см., например, [8])

$$
\nu_{1}(x)=\frac{1}{\Gamma\left(1+\alpha_{0}\right)} \tau_{1}^{\prime \prime}(x)
$$

Найдем функциональное соотношение между $\tau_{2}(x)$ и $\nu_{2}(x)$, принесенное на линию $y=0$ из гиперболической части $\Omega_{2}$ области $\Omega$.

Используя формулу решения задачи Коши в области $\Omega_{2}$, выпишем $u\left[\Theta_{0}(x)\right]$ :

$$
u\left[\Theta_{0}(x)\right]=k_{1}\left(I_{0+}^{\alpha, 0, \beta-1} \tau_{2}(t)\right)(x)+k_{2}\left(I_{0+}^{1-\beta, \alpha+\beta-1, \beta-1} \nu_{2}(t)\right)(x),
$$

где

$$
k_{1}=\frac{\Gamma(\alpha+\beta)}{\Gamma(\beta)}, \quad k_{2}=\frac{\Gamma(2-\alpha-\beta)}{\Gamma(1-\alpha)}\left(\frac{m+2}{4}\right)^{\frac{2}{m+2}} .
$$


Подставляя $u\left[\Theta_{0}(x)\right]$ в краевое условие $(3)$ и используя полугрупповое свойство обобщенных операторов [1, с. 327]

$$
I_{0+}^{\alpha, \beta, \eta} I_{0+}^{\gamma, \delta, \alpha+\eta} f=I_{0+}^{\alpha+\gamma, \beta+\delta, \eta} f, \quad \gamma>0
$$

получим

$$
\begin{aligned}
& k_{1} A\left(I_{0+}^{a+\alpha, b, \beta-1-a} \tau_{2}(t)\right)(x)+k_{2} A\left(I_{0+}^{a+1-\beta, b+\alpha+\beta-1, \beta-1-a} \nu_{2}(t)\right)(x)+ \\
& +B\left(I_{0+}^{a_{1}, a+b+\alpha-a_{1}, \beta-1-a} \nu_{2}(t)\right)(x)+C\left(I_{0+}^{a_{2}, a+b+\alpha-a_{2}, \beta-1-a} \nu_{2}(t)\right)(x)=g(x) .
\end{aligned}
$$

Подействуем на обе части соотношения (6) оператором $I_{0+}^{-a-\alpha,-b, \alpha+\beta-1}$. Непосредственные вычисления с использованием формулы (5) дают возможность записать

$$
\begin{aligned}
\tau_{2}(x)=k_{3}\left(I_{0+}^{1-\alpha-\beta} \nu_{2}(t)\right)(x)+k_{4}\left(I_{0+}^{a_{1}-a-\alpha} \nu_{2}(t)\right)(x)+ \\
+k_{5}\left(I_{0+}^{a_{2}-a-\alpha} \nu_{2}(t)\right)(x)+g_{1}(x),
\end{aligned}
$$

где $I_{0+}^{\alpha}$ - оператор Римана-Лиувилля,

$$
k_{3}=-\frac{k_{2}}{k_{1}}, \quad k_{4}=-\frac{B}{A k_{1}}, \quad k_{5}=-\frac{C}{A k_{1}} ; \quad g_{1}(x)=\frac{1}{A k_{1}}\left(I_{0+}^{-a-\alpha,-b, \alpha+\beta-1} g(t)\right)(x) .
$$

При $g(x)=0$ покажем, что интеграл

$$
I=\int_{0}^{1} \tau_{2}(x) \nu_{2}(x) d x \geqslant 0 .
$$

В самом деле, опираясь на (7), имеем

$$
\begin{aligned}
& I=\frac{k_{3}}{\Gamma(1-\alpha-\beta)} \int_{0}^{1} \nu_{2}(x) d x \int_{0}^{x} \nu_{2}(t)(x-t)^{-\alpha-\beta} d t+ \\
& \quad+\frac{k_{4}}{\Gamma\left(a_{1}-a-\alpha\right)} \int_{0}^{1} \nu_{2}(x) d x \int_{0}^{x} \nu_{2}(t)(x-t)^{a_{1}-a-\alpha-1} d t+ \\
& \quad+\frac{k_{5}}{\Gamma\left(a_{2}-a-\alpha\right)} \int_{0}^{1} \nu_{2}(x) d x \int_{0}^{x} \nu_{2}(t)(x-t)^{a_{2}-a-\alpha-1} d t .
\end{aligned}
$$

Далее, проводя стандартные вычисления (см., например, [9]), получим $I \geqslant 0$, а для области $\Omega_{1}-I \leqslant 0$. Из этих неравенств имеем $I \equiv 0$. Последнее тождество влечет за собой выполнение тождества $u(x, y) \equiv 0$, что и доказывает единственность решения исходной задачи.

3. Существование решения задачи. Для доказательства существования решения исследуемой задачи достаточно найти $\nu_{1}(x)$ или $\nu_{2}(x)$.

Полагая $\tau_{1}=\tau_{2}(x)=\tau(x), \nu_{1}(x)=\nu_{2}(x)=\nu(x)$, продифференцируем обе части соотношения $(7)$ дважды по $x$. Получим уравнение

$$
\begin{aligned}
\tau^{\prime \prime}(x)=k_{3}\left(D_{0+}^{1+2 \beta} \nu\right)(x)+k_{4}\left(D_{0+}^{2+a+\alpha-a_{1}} \nu\right) & (x)+ \\
& +k_{5}\left(D_{0+}^{2+a+\alpha-a_{2}} \nu\right)(x)+g_{1}^{\prime \prime}(x) .
\end{aligned}
$$


Здесь $\left(D_{0+}^{\alpha} f\right)(x)$ - дробная производная в смысле Римана-Лиувилля.

В силу (4)

$$
\tau^{\prime \prime}(x)=\Gamma\left(1+\alpha_{0}\right) \nu(x),
$$

тогда уравнение (8) принимает вид

$$
\left(D_{0+}^{1+2 \beta} \nu\right)(x)-\lambda\left(D_{0+}^{2+a+\alpha-a_{1}} \nu\right)(x)-\delta\left(D_{0+}^{2+a+\alpha-a_{2}} \nu\right)(x)-\mu \nu(x)=g_{2}(x),
$$

где

$$
\lambda=-\frac{k_{4}}{k_{3}}, \quad \delta=-\frac{k_{5}}{k_{3}}, \quad \mu=-\frac{\Gamma\left(1+\alpha_{0}\right)}{k_{3}}, \quad g_{2}(x)=-\frac{1}{k_{3}} g_{1}^{\prime \prime}(x) .
$$

В монографии [10, с. 301] рассмотрено уравнение дробного порядка

$$
\left(D_{0+}^{\alpha} y\right)(x)-\lambda\left(D_{0+}^{\beta} y\right)(x)-\delta\left(D_{0+}^{\gamma} y\right)(x)=f(x) \quad(x>0, \lambda, \delta, \mu \in \mathbb{R}),
$$

где

$$
l-1<\alpha \leqslant l \quad(l \in \mathbb{R}), \quad 0<\gamma<\beta<\alpha,
$$

и выписано его решение в виде

$$
\begin{gathered}
y(x)=\int_{0}^{x}(x-t)^{\alpha-1} G_{\gamma, \beta, \alpha, \lambda}(x-t) f(t) d t \\
G_{\gamma, \beta, \alpha, \lambda}(z)=\sum_{n=0}^{\infty}\left(\sum_{i+v=n}\right) \frac{\mu^{i} \delta^{v}}{i ! v !} z^{(\alpha-\beta) n+\beta i-(\beta-\gamma) v} \times \\
\times{ }_{1} \Psi_{1}\left[\begin{array}{l}
(n+1,1) \\
((\alpha-\beta) n+\beta i+\alpha+(\beta-\gamma) v, \alpha-\beta)
\end{array} \mid \lambda z^{\alpha-\beta}\right] .
\end{gathered}
$$

Для уравнения (9) это решение принимает вид

$$
\nu(x)=\int_{0}^{x}(x-t)^{2 \beta} G_{2+a+\alpha-a_{2}, 2+a+\alpha-a_{1}, 1+2 \beta, \lambda}(x-t) g_{2}(t) d t
$$

где

$$
\begin{aligned}
& G_{2+a+\alpha-a_{2}, 2+a+\alpha-a_{1}, 1+2 \beta, \lambda}(x)= \\
& =\sum_{n=0}^{\infty}\left(\sum_{i+v=n}\right) \frac{\mu^{i} \delta^{v}}{i ! v !} z^{\ell}{ }_{1} \Psi_{1}\left[\begin{array}{c}
(n+1,1) \\
\left(\ell, a_{1}+2 \beta-a-\alpha\right)
\end{array} \mid \lambda z^{a_{1}+2 \beta-1-a-\alpha}\right] \\
& \ell=\left(a_{1}+2 \beta+1-a+\alpha\right) n+\left(2+a+\alpha-a_{1}\right) i-\left(a_{2}-a_{1}\right) v .
\end{aligned}
$$

Поскольку решение исследуемой задачи построено в явном виде, существование решения можно считать доказанным.

Декларация о финансовых и других взаимоотношениях. Исследование не имело спонсорской поддержки. Все авторы принимали участие в разработке концепции статьи и в написании рукописи. Авторы несут полную ответственность за предоставление окончательной рукописи в печать. Окончательная версия рукописи была одобрена всеми авторами. Авторы не получали гонорар за статью. 


\section{ORCIDs}

Олег Александрович Репин: http://orcid.org/0000-0003-1522-3955

Анна Валерьевна Тарасенко: http://orcid.org/0000-0002-0487-8262

\section{БИБЛИОГРАФИЧЕСКИЙ СПИСОК}

1. Самко С. Г., Килбас А. А., Маричев О. И. Интеграль и производные дробного порядка и некоторые их приложения. Минск: Наука и техника, 1987. 688 с.

2. Saigo M. A remark on integral operators involving the Gauss hypergeometric function // Math. Rep. Coll. Gen. Educ., Kyushu Univ., 1978. vol.11, no.2. pp. 135-143, http:// catalog.lib.kyushu-u.ac.jp/handle/2324/1449009/11_2_p135.pdf.

3. Репин О. А. Краевые задачи со смещением для уравнений гиперболического и смешанного типов. Саратов: Саратов. ун-т, 1992. 161 с.

4. Нахушев А. М. Дробное исчисление его применение. М.: Физматлит, 2009. 272 с.

5. Килбас А. А., Репин О. А. Аналог задачи Бицадзе-Самарского для уравнения смешанного типа с дробной производной // Дифферени. уравнения, 2003. Т. 39, № 5. С. 638-644.

6. Тарасенко А. В. О разрешимости нелокальной задачи для нагруженного парабологиперболического уравнения // Изв. вузов. Матем., 2013. № 1. С. 73-81.

7. Псху А. В. Уравнения в частных производных дробного порядка. М.: Наука, 2005. 199 с.

8. Килбас А. А., Репин О. А. О разрешимости краевой задачи для уравнения смешанного типа с частной дробной производной Римана-Лиувилля // Дифференц. уравнения, 2010. Т. 46, № 10. С. 1453-1460.

9. Репин О. А. Краевая задача для дифференциального уравнения с частной дробной производной Римана-Лиувилля // Уфимск. матем. журн., 2015. Т. 7, № 3. С. 70-75.

10. Kilbas A. A., Srivastava H. M., Trujillo Y. Y. Theory and Applications of Fractional Differential Equations / North-Holland Mathematics Studies. vol. 204 / ed. J. van Mill. Amsterdam: Elsevier, 2006. 523 pp.

Поступила в редакцию $25 / \mathrm{X} / 2016$;

в окончательном варианте - 14/XI/2016;

принята в печать - 09/XII/2016. 
Vestn. Samar. Gos. Techn. Un-ta. Ser. Fiz.-mat. nauki

[J. Samara State Tech. Univ., Ser. Phys. \& Math. Sci.], 2016, vol. 20, no. 4, pp. 636-643

ISSN: 2310-7081 (online), 1991-8615 (print)

doi: http://dx.doi.org/10.14498/vsgtu1516

MSC: 35M12

\title{
ON A PROBLEM FOR MIXED TYPE EQUATION WITH PARTIAL RIEMANN-LIOUVILLE FRACTIONAL DERIVATIVE
}

\author{
O. A. Repin, A. V. Tarasenko \\ 1 Samara State Economic University, \\ 141, Sovetskoy Armii st., Samara, 443090, Russian Federation. \\ 2 Samara State Technical University, \\ 244, Molodogvardeyskaya st., Samara, 443100, Russian Federation.
}

\begin{abstract}
The uniqueness and existence of solutions of a nonlocal problem proved for an equation of mixed type. This equation contains diffusion equation of fractional order. The boundary condition contains a linear combination of generalized operators of fractional order with the Gauss hypergeometric function. The solution of the problem is given explicitly.
\end{abstract}

Keywords: boundary value problem, generalized fractional integro-differentiation operator, differential equation of fractional order, Gauss hypergeometric function.

Declaration of Financial and Other Relationships. The research has not had any sponsorship. Each author has participated in the article concept development and in the manuscript writing. The authors are absolutely responsible for submitting the final manuscript in print. Each author has approved the final version of manuscript. The authors have not received any fee for the article.

\section{ORCIDs}

Oleg A. Repin: http://orcid.org/0000-0003-1522-3955

Anna V. Tarasenko: http://orcid.org/0000-0002-0487-8262

\section{REFERENCES}

1. Samko S. G., Kilbas A. A., Marichev O. I. Integraly i proizvodnye drobnogo poriadka $i$ nekotorye ikh prilozheniia [Integrals and derivatives of fractional order and some of their applications]. Minsk, Nauka i Tekhnika, 1987, 688 pp. (In Russian)

(C) 2016 Samara State Technical University.

Please cite this article in press as:

Repin O. A., Tar a s en k o A. V. On a problem for mixed type equation with partial RiemannLiouville fractional derivative, Vestn. Samar. Gos. Tekhn. Univ., Ser. Fiz.-Mat. Nauki [J. Samara State Tech. Univ., Ser. Phys. \& Math. Sci.], 2016, vol. 20, no. 4, pp. 636-643. doi: 10.14498/vsgtu1516. (In Russian)

Authors Details:

Oleg A. Repin (Dr. Phys. \& Math. Sci.; Professor; matstat@mail.ru), Head of Dept., Dept. of Mathematical Statistics and Econometrics ${ }^{1}$; Professor, Dept. of Applied Mathematics \& Computer Science ${ }^{2}$.

Anna V. Tarasenko (Cand. Phys. \& Math. Sci.; tarasenko.a.v@mail.ru; Corresponding Author), Associate Professor, Dept. of Higher Mathematics of the Architectural Engineering Institute. 
2. Saigo M. A remark on integral operators involving the Gauss hypergeometric function, Math. Rep. Coll. Gen. Educ., Kyushu Univ., 1978, vol.11, no. 2, pp. 135-143, http://catalog. lib.kyushu-u.ac.jp/handle/2324/1449009/11_2_p135.pdf.

3. Repin O. A. Kraevye zadachi so smeshcheniem dlia uravnenii giperbolicheskogo $i$ smeshannogo tipov [Boundary value problems with a shift for hyperbolic and mixed type equations]. Saratov, Saratov. Univ., 1992, 161 pp. (In Russian)

4. Nakhushev A. M. Drobnoe ischislenie ego primenenie [Fractional calculus and its applications]. Moscow, Fizmatlit, 2009, 272 pp. (In Russian)

5. Kilbas A. A., Repin O. A. An Analog of the Bitsadze-Samarskii Problem for a Mixed Type Equation with a Fractional Derivative, Differ. Equ., 2003, vol. 39, no. 5, pp. 674-680. doi: $10.1023 / \mathrm{A}: 1026194020442$.

6. Tarasenko A. V. Solvability of a nonlocal problem for a loaded parabolic-hyperbolic equation, Russian Math. (Iz. VUZ), 2013, vol.57, no.1, pp. 64-71. doi:10.3103/ S1066369X13010076.

7. Pskhu A. V. Uravneniia v chastnykh proizvodnykh drobnogo poriadka [Partial differential equations of fractional order]. Moscow, Nauka, 2005, 199 pp.

8. Kilbas A. A., Repin O. A. Solvability of a boundary value problem for a mixed-type equation with a partial Riemann-Liouville fractional derivative, Differ. Equ., 2010, vol. 46, no. 10, pp. 1457-1464. doi: 10.1134/S0012266110100095.

9. Repin O. A. Boundary value problem for partial differential equation with fractional Riemann-Liouville derivative, Ufa Math. Journal, 2015, vol.7, no. 3, pp. 67-72. doi: 10. 13108/2015-7-3-67.

10. Kilbas A. A., Srivastava H. M., Trujillo Y. Y. Theory and Applications of Fractional Differential Equations, North-Holland Mathematics Studies, vol. 204, ed. J. van Mill. Amsterdam, Elsevier, 2006, 523 pp.

Received 25/X/2016;

received in revised form $14 / \mathrm{XI} / 2016$;

accepted 09/XII/2016. 